Klaus Müller*

\title{
Riester-Reform - Nur eine Erleichterung für die Anbieter
}

https://doi.org/10.1515/zfwp-2020-2043

\begin{abstract}
The Riester pension was well thought out, but badly done. It is primarily the agents and insurance companies who benefit from Riester, while consumers hardly benefit at all - and if they do, it is only because of the generous state subsidy. The reform proposals to date do not eliminate the deficits, but are rather a restructuring plan for the supply side. However, private pension contracts should also pay off without subsidies. State-organised standard products in Sweden or Great Britain show how such efficiency can be achieved. The German "Verbraucherzentrale Bundesverband" has made a suggestion in this respect, the "Extrarente": a public body pools consumer demand and can thus cost-effectively organise their capital investment on a share scheme basis.
\end{abstract}

Walter Riester hat es gut gemeint. Im November 2000 verkündete der damalige SPD-Arbeitsminister im Deutschen Bundestag, mit der nach ihm benannten Rentenreform „das Rentenniveau insgesamt dauerhaft anzuheben“. 20 Jahre später ist klar: Riesters Experiment, die private Altersvorsorg in die Hände der Versicherungswirtschaft zu geben, ist nicht gelungen. Das Rentenniveau ist nicht gestiegen, sondern gefallen. Viele Riester-Produkte sind zu teuer, zu unübersichtlich und zu ineffizient. Es profitieren vor allem Versicherer und Finanzvertriebe. Und würde der Staat nicht Milliarden aus Steuermitteln hinzuschießen, käme für Verbraucher noch weniger dabei rum.

\section{Wo liegen die derzeitigen Probleme?}

Verbraucherinnen und Verbraucher ${ }^{1}$, die ihren Lebensstandard auch im Ruhestand aufrecht erhalten wollen, sind auf eine Zusatzvorsorge über den Kapitalmarkt angewiesen. Mit der Einführung der Riester-Rente hat die Politik das

1 Die im weiteren Text gewählte männliche Form bezieht sich immer zugleich auf Personen aller

*Kontakt: Klaus Müller, Verbraucherzentrale Bundesverband e.V., Rudi-Dutschke-Str.17, 10969 Berlin 
Sicherungsniveau der Gesetzlichen Rentenversicherung (GRV) dann schrittweise abgesenkt und gleichzeitig die private Vorsorge im Rahmen des sogenannten DreiSäulen-Modells zum freiwilligen aber expliziten Bestandteil der Alterssicherung erklärt. $^{2}$

Für viele Menschen scheitert die zusätzliche Altersvorsorge am fehlenden Geld. Immer mehr Verbraucher sind nicht in der Lage, ausreichend Ersparnisse zu bilden. Die Schere zwischen Vermögenden und nicht Vermögenden geht immer weiter auseinander. Das führt dazu, dass Wohlhabende durchaus „riestern“ und von der staatlichen Förderung profitieren - weniger Wohlhabende demgegenüber nicht oder deutlich weniger. Das ursprüngliche Ziel, Verbrauchern Anreize zu neuen Sparanstrengungen zu geben, wird also verfehlt. ${ }^{3}$

Aber auch alle, die Geld haben, um es in Riesterprodukte anzulegen, haben ein Problem: Die Vorsorgeprodukte sind komplex und der Markt ein Verkäufermarkt, auf dem teure Produkte durch Banken, Versicherungen und Vermittler provisionsgesteuert verkauft werden.

Die Fakten sind klar: Riester-Verträge rechnen sich in aller Regel nur durch die staatliche Förderung. So titelte die Stiftung Warentest in ihrem letzten produktübergreifenden Riester-Test aus dem Jahr 2017 treffend: „Nur mit Geld vom Staat“. Tatsächlich betrugen die Ausgaben für die Förderung von bestehenden Verträgen im Jahr 2018 insgesamt gut 3,8 Milliarden Euro. ${ }^{4}$

Die Förderung jedoch verhindert einen echten Produktwettbewerb und wird in der Praxis einseitig zur Absatzförderung genutzt. Heißt: Banken, Versicherer und Vermittler versprechen ihren Kunden mit Verweis auf die Zulagen attraktive Renditen - und verschweigen meist die offensichtlichen Schwächen der Produkte wie geringe Flexibilität, hohe Kosten und die Tatsache, dass die Zulagenförderung beziehungsweise der ergänzende Steuerabzug nur ein Teil der Rechnung ist. Die Riester-Rente muss im Alter voll versteuert werden. Ohne Förderung und vorherigen Steuerabzug wäre sie für Verbraucher ein Minusgeschäft.

Über den Erfolg eines Produktes entscheidet so nicht dessen Qualität, sondern seine Attraktivität für Vertriebe.

Geschlechter. Wir bitten um Verständnis für den weitgehenden Verzicht auf Mehrfachbezeichnungen zugunsten einer besseren Lesbarkeit des Textes.

2 vgl. Mohn, Ahlers, Private Zusatzvorsorge neu denken: Deutschland braucht ein Standardprodukt, Vierteljahrshefte zur Wirtschaftsforschung 2019, S. 113-123.

3 vgl. ebenda.

4 vgl. Verbraucherzentrale Bundesverband, Riester-Reform gefährdet Altersvorsorge, https:// www.vzbv.de/sites/default/files/downloads/2020/10/02/fin-20-10-02_riester-reform-gefaehrdetaltersvorsorge2-br.pdf. 
Da sich die Provisionen als Kosten im Produkt verstecken, führt ein solcher „Wettbewerb der Vertriebe“ dazu, dass sich Produkte mit hohen Kosten am Markt eher durchsetzen, als Produkte mit niedrigen Kosten. Hohe Kosten gehen dabei jedes Jahr von der Rendite ab und schmälern so den Wert der Anlage und letztlich die Höhe der Renten. Die eigentlich als Kaufanreiz gedachte Zulagenförderung dient so vielfach nur noch dazu, die hohe Kostenbelastung auszugleichen und erzeugt eine Scheinrentabilität.

Die Wahrheit ist, dass die staatlich geförderte Riester-Rente nicht funktioniert. Sie ist intransparent, teuer und Rendite lässt sich meist überhaupt nur durch staatliche Zulagen erzielen.

\section{Reformvorwünsch der Versicherungsbranche sind kein Ausweg aus dem Dilemma sie würden das Dilemma verschlimmern}

In ihrem Koalitionsvertrag hat die Bundesregierung daher angekündigt, die private Altersvorsorge $\mathrm{zu}$ verbessern und einfacher $\mathrm{zu}$ gestalten. Dazu soll ein attraktives standardisiertes Riester-Produkt geschaffen werden. Die aktuell diskutierten Vorschläge zur Reform der Riester-Rente greifen weder den Koalitionsvertrag auf, noch sind sie geeignet, die aufgezeigten Probleme zu lösen und die Altersvorsorge zu verbessern. ${ }^{5}$

\section{Produktstandard und mehr Förderung}

Ins Feld geführt wird ein so genannter Produktstandard. Der Gesetzgeber definiert Kriterien für ein standardisiertes Produkt, dass jeder Riester-Anbieter dann neben den sonstigen, weniger regulierten Marktprodukten stellen kann. Das kann nicht funktionieren. Die Reglementierung, insbesondere ein - wie auch immer gearteter - Kostendeckel wird den Vertrieb dazu veranlassen, von der Standardvariante weg zu beraten und den Kauf der anderen Variante zu empfehlen. Der Vertrieb hat immer den Anreiz, das höher provisionierte Produkt zu verkaufen. Dies hat die Einführung der Riester-Rente gezeigt. Das Schlagwort „Riester“ war der Türöffner für das Verkaufsgespräch, in dessen Verlauf die vermeintlichen

5 ebenda. 
Vorzüge der ungeförderten Variante angepriesen wurden. Diesmal wäre der Türöffner eine Erhöhung der Zulagenförderung auf 40 Prozent und eine einheitliche Kinderzulage von 300 Euro.

\section{Absenkung der Beitragserhaltungszusagen}

Derzeit müssen Anbieter von Riester-Produkten vertraglich zusagen, dass zu Beginn zu Rentenbeginn mindestens die eingezahlten Beiträge vorhanden sind. Die Anbieter fordern eine Absenkung dieser so genannten Beitragserhaltungszusage. Vorgeblich um die Kapitalanlage zugunsten der Verbraucher anpassen zu können.

Die Aufweichung der Beitragserhaltungszusage hat aber noch eine ganz andere Folge. Bisher begrenzt die Beitragserhaltungszusage bei Riester-Verträgen die in ein Riesterprodukt hineingerechneten Kosten. In der derzeitigen Niedrigzinsphase stellt die Beitragserhaltungszusage ein erhebliches Problem für Produktanbieter dar. Durch die fallenden Renditen können hohe Kosten nicht mehr so einfach ausgeglichen werden. Die Reaktion der Anbieter ist, das Angebot gerade für kurze Laufzeiten einzustellen.

Eine Absenkung der Beitragserhaltungsgarantie ermöglicht es den Anbietern, bei kürzeren Laufzeiten die Kosten nicht senken $\mathrm{zu}$ müssen und bei längeren Laufzeiten sogar höhere Kosten einzukalkulieren. Eine Untersuchung der Stiftung Warentest hat bei nichtgeförderten Rentenversicherungen festgestellt, dass Verträge mit geringerem Garantieniveau teurer waren als Verträge mit hohen Garantien. ${ }^{6}$ Anbieter nutzen also die höhere Freiheit durch eine verminderte Garantie tatsächlich nicht primär dazu, höhere Rendite für Verbraucher $z u$ erzielen, sondern höhere Kosten einzupreisen.

\section{Kostendeckel}

Man gibt sich weiter verbraucherfreundlich und führt die Idee eines Kostendeckels ins Feld. Allerdings hat dieser Kostendeckel, der bei einem Prozent Effektivkosten liegen soll, mehrere Haken. So soll der Kostendeckel lediglich für die Standardvariante gelten, woraus wiederrum das Wettbewerbsproblem $\mathrm{zu}$ den restlichen Riester-Angeboten im Raum steht.

6 vgl. Stiftung Warentest, Vorsorge für Methusalem, Finanztest 2019, S. $42-45$. 
Daneben hört sich ein Prozent Renditeminderung durch Kosten wenig an kann für Verbraucher aber richtig teuer sein. Um dies zu verstehen, müssen wir auf die Definition der Renditeminderung durch Kosten blicken. Sie drücken den Verlust an Rendite aus, der durch vorhandene Kosten - zum Beispiel Verwaltungskosten - über die gesamte Laufzeit entsteht. Bezieht man diese Kosten jedoch auf den gezahlten Beitrag, entsteht ein ganz anderes Bild: Nimmt man zum Beispiel eine Laufzeit von 30 Jahren und eine unterstellte Rendite von vier Prozent an, liegen bei einem Prozent Effektivkosten die Kosten bezogen auf den Beitrag bei 15 Prozent. Von 100 Euro, die Verbraucher einzahlen, kommen also nur 85 Euro in den Spartopf. Bei einer angenommenen Rendite von zwei Prozent lägen die Kosten sogar bei 21 Prozent. $^{7}$

\section{Fazit}

Die Bestandsaufnahme hat gezeigt: Die Riester-Rente war gut gedacht, aber schlecht gemacht. Von Riester profitieren vor allem die Vermittler und Versicherer, Verbraucher dagegen kaum - und wenn, dann nur aufgrund der großzügigen staatlichen Förderung. Die bisherigen Reformvorschläge wiederum beseitigen nicht die Defizite, sondern sind eher ein Sanierungsprogramm für die Anbieterseite.

Hier würde noch mehr Geld in die Hand genommen, um ineffiziente Produkte mit Steuergeldern zu subventionieren. Private Vorsorgeverträge sollten sich aber auch ohne Subventionen rechnen. Staatlich organisierte Standardprodukte in Schweden oder Großbritannien machen vor, wie eine solche Effizienz erzielt werden kann. Daran sollte sich die Bundesregierung orientieren. Hierzu hat der Verbraucherzentrale Bundesverband einen Vorschlag gemacht, die Extrarente: ein öffentlicher Träger bündelt die Nachfrage der Verbraucher und kann so kosteneffizient deren Kapitalanlage auf Aktienbasis organisieren.

Dann wäre die Zulagenförderung ein „Sahnehäubchen“ für diejenigen, die private Altersvorsorge betreiben können. Wer Altersarmut vermeiden will, muss diejenigen gezielt unterstützen, die nicht oder nur wenig Erspartes haben. Eine denkbare Alternative zum bisherigen System wäre etwa, die Förderung in die gesetzliche Rentenversicherung einzuzahlen.

7 vgl. Verbraucherzentrale Bundesverband, Riester-Reform gefährdet Altersvorsorge, https:// www.vzbv.de/sites/default/files/downloads/2020/10/02/fin-20-10-02_riester-reform-gefaehrdetaltersvorsorge2-br.pdf. 